\title{
Association of Mycobacterium Leprae with Human Endothelial Cells In Vitro
}

\author{
David M. Scollard \\ Department of Research Pathology, GWL Hansen's Disease Center, and Department of Pathology, LSU School of \\ Medicine, New Orleans, Louisiana
}

SUMMARY: Endothelial cell infection by Mycobacterium leprae has long been described histologically in all types of leprosy and in some of the acute reactions occurring in this disease. Recent evidence from experimental lepromatous neuritis indicates that $M$. leprae colonizes endothelial cells of epineural blood vessels even in sites of minimal infection, suggesting that interaction between these cells and $M$. leprae may play an important role in the selective localization of this organism to peripheral nerve. To begin to study the mechanisms involved, we have examined the interaction between $M$. leprae and human umbilical vein endothelial cells (HUVEC) in vitro using light microscopy, scanning and transmission electron microscopy, and confocal laser scanning microscopy. When $M$. leprae were added to confluent monolayers of HUVEC, uptake increased slowly to a maximum at 24 hours. Maximal percentages of infected cells were similar at ratios of organisms:cell over a range of 25:1 to 100:1. The bacilli appeared to lie within membrane-bound vacuoles at all time points. The kinetics of association of $M$. leprae with HUVEC are much slower than has previously been observed with macrophages, possibly due to differences in the binding of $M$. leprae. Compared with other pathogens that infect endothelial cells, $M$. leprae also appear to be ingested more slowly, and to a more limited degree. The receptors involved in $M$. leprae binding to endothelial cells and the impact of intracellular infection by $M$. leprae on these cells remain to be determined. (Lab Invest 2000, 80:663-669).

\begin{abstract}
Infection of endothelial cells (EC) by Mycobacte1 rium leprae was repeatedly noted in the early histopathologic descriptions of leprosy (Hansen and Looft, 1895; Klingmüller, 1930). The extent of EC infection in this disease was reported in detail in a large study by Fite in 1941, who noted focal infection of blood vessels in $42 \%$ of skin lesions of all types, "usually with bacilli in the lining endothelial cells." Later studies further described EC infection by $M$. leprae at the light microscopic and ultrastructural levels (Boddingius, 1984; Burchard and Bierther, 1985; Coruh and McDougall, 1979; Fite, 1941; Mukherjee et al, 1987; Turkel et al, 1982). The remarkable extent of these descriptions, compared with other diseases, was emphasized in a review of the phagocytic properties of EC (Ryan, 1988). The pathogenesis of leprosy is now widely understood from the perspective of its unique immunologic spectrum, and EC involvement has not been widely appreciated among students of leprosy. However, the current cellular and molecular knowledge of immunologic mechanisms in leprosy do not obviate the longstanding observation of EC infection; rather, infection of EC appears to have been largely overlooked because, for over four decades, it had no apparent role in the immunological issues that dominated research on leprosy. Recent observations on the localization of $M$. leprae to peripheral nerves have indicated that EC play an important
\end{abstract}

Received December 2, 1999.

Address reprint requests to: Dr. D. M. Scollard, GWL Hansen's Disease Center at LSU, P.O. Box 25072, Baton Rouge, Louisiana 70894. Fax: 225-346-5786; E-mail: dscoll1@.lsu.edu role in this process (Scollard et al, 1996, 1999). These studies provide the most direct evidence to date that the vasculature plays a central role in the unique tropism of $M$. leprae to peripheral nerves, and has given new impetus to the study of the interaction between this pathogen and EC. The culture of EC isolated from human umbilical veins has become an established model for research into many aspects of EC biology (Gimbrone, 1976), including infection with other micro-organisms such as Listeria (Drevets et al, 1995; Greiffenberg et al, 1998), Candida (Filler et al, 1995; Fratti et al, 1998), and Rickettsiae (Austin and Winkler, 1988; Walker, 1984). We have therefore examined the ability of HUVEC to ingest $M$. leprae under controlled conditions in vitro, and documented basic aspects of this interaction by light, electron, and confocal microscopy.

\section{Results}

$M$. leprae were associated with a only a small percentage of HUVEC after 3 or 6 hours of co-cultivation, but the percentage of HUVEC associated with bacilli increased to a maximum at approximately 24 hours (Fig. 1A). After 24 hours, the percentage of infected cells appeared to decline slightly.

At a multiplicity of infection (MOI) of 100:1 or less, approximately $50 \%$ of cells had bound and/or ingested bacilli at 24 hours (Fig. 1B). However, $>95 \%$ of HUVEC were associated with $M$. leprae at $\mathrm{MOI}$ of 1000:1 at 24 hours.

Study of only those cells that were infected at each time point, at $\mathrm{MOI}$ of $100: 1$ or less, revealed that the 

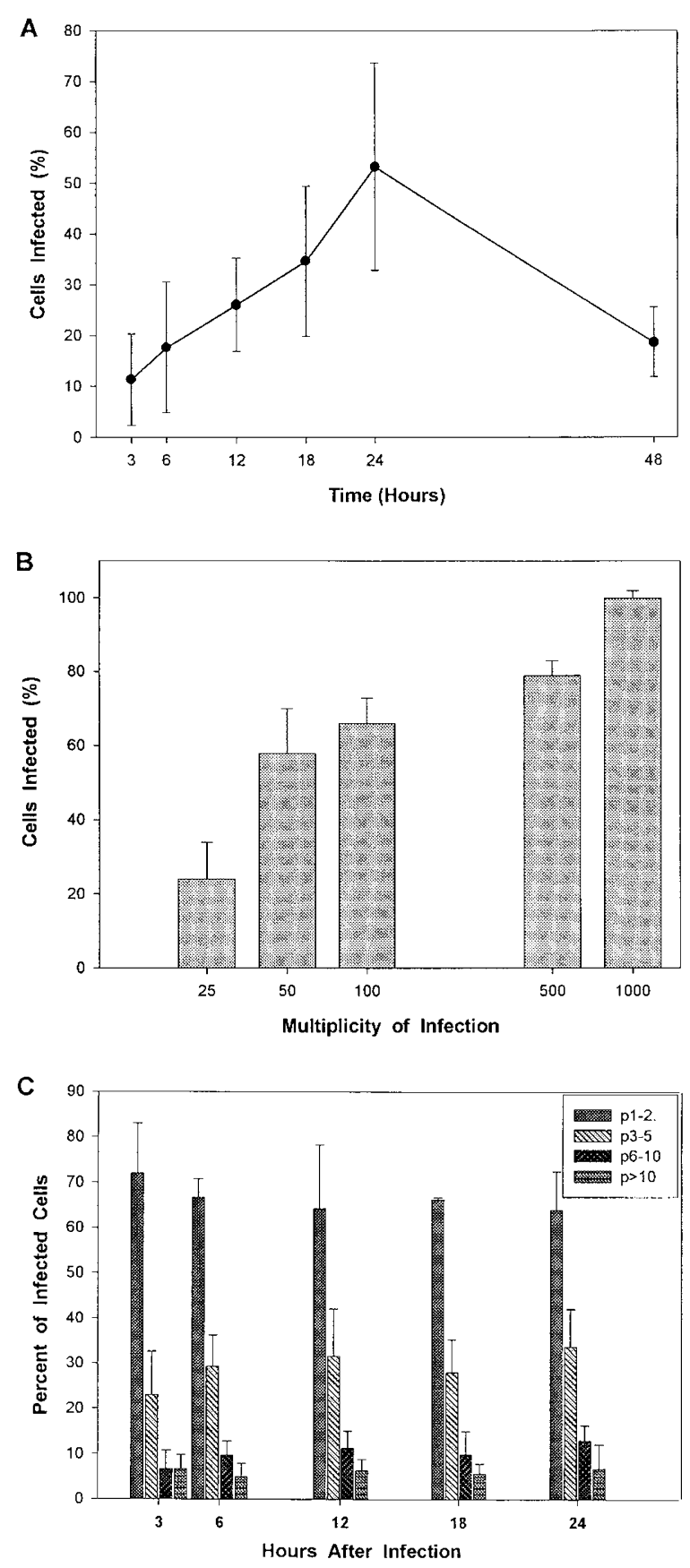

Figure 1.

Human umbilical vein endothelial cells (HUVEC) ingest $M$. leprae in a time- and concentration-dependant manner. A, Bacilli at a multiplicity of infection (MOI) of 100:1 were associated with a small percentage of cells after 3 or 6 hours exposure, increasing to a maximum at 24 hours. Mean $+/$ - SD of four experiments. B, Saturation of HUVEC with $M$. leprae occurred only at a high $\mathrm{MOI}$. Bars indicate the mean $+/$ - SD of three experiments. The number of bacilli bound and/or ingested only reached approximately $60 \%$ at MOI of $100: 1$. However, at MOI of 1000:1, $>95 \%$ of HUVEC were associated with M. leprae. $\mathrm{C}$, The number of bacilli per infected cell was similar at all time points. Bars indicate the mean $+/$ - SD of three experiments. The number of bacilli per cell, at MOI of 100:1, was determined for 100 infected cells, scored as 1 to 2 bacilli/cell, 3 to 5 bacilli/cell, 6 to 10 bacilli/cell, and $>10$ bacilli/cell.

number of bacilli per infected EC was similar at all time points. Most of the infected cells contained only a small number of bacilli, and only a small fraction of them contained $>5$ bacilli (Fig. 1C). Notably, this distribution of bacilli within infected cells was consistent over at least the first 24 hours of co-cultivation of HUVEC with $M$. leprae, even though the percentage of cells that were infected increased during this interval (Fig. 1A).

By scanning electron microscopy (SEM), the binding of M. leprae to HUVEC was apparent within 1 hour, and bacilli could be seen attached to the cell surface of HUVEC for at least the first 24 hours (Fig. 2). Adherent bacilli were not quantified, but inspection of (SEM) preparations of cultures from 3 to 24 hours did not indicate any substantial increase or decrease in the number of bacilli bound to the cell surface.

Confocal laser scanning microscopy of 24-hour cultures confirmed the surface location of some bacilli, appearing as green (auramine-O-positive) points at the same level as the red (UEA-1-positive) HUVEC cell membrane (Fig. 3B). Most organisms, however, had been internalized and were located below the cell membrane.

Transmission electron microscopy revealed that organisms were located inside membrane-bound phagocytic vacuoles as early as 1 hour after addition of M. leprae to HUVEC (Fig. 4, A and B). This intracellular location of $M$. leprae within phagocytic vacuoles was observed throughout 24 hours of observation (Fig. 4, C and D).

\section{Discussion}

These results demonstrate that $M$. leprae are bound and ingested by EC in vitro, but that this process occurs more slowly than has been observed with professional phagocytes (Sibley et al, 1987). In addition, a very high $\mathrm{MOI}$ is necessary to achieve saturation of HUVEC, compared with professional phagocytes in which nearly $100 \%$ contain M. leprae after exposure to a lower $\mathrm{MOI}$.

When the duration of co-culture of HUVEC and $M$. leprae was extended over 24 hours, the distribution of bacilli per cell remained relatively constant, with only a small fraction of cells ingesting a large number of bacilli at any time point. This suggests that most HUVEC will not continue to ingest large numbers of $M$. leprae even if more are available, but appear to abstain from further phagocytosis. This could be a function of the number of viable bacilli, as with phagocytosis of rickettsiae (reviewed in Austin and Winkler, 1988), but at this time there is no means by which to accurately determine the viability of $M$. leprae.

The rate-limiting step in uptake may be slower phagocytosis or reduced adherence to EC due to lower frequency or slower expression of binding molecules on EC. The observation of $M$. leprae intracellularly at 1 and 3 hours, however, indicates that the mechanics of phagocytosis may not be delayed, and therefore suggests that binding is likely to be the rate-limiting step.

Taken together, these results suggest that at $\mathrm{MOI}$ of 100:1, only a small percentage of HUVEC bind $M$. 

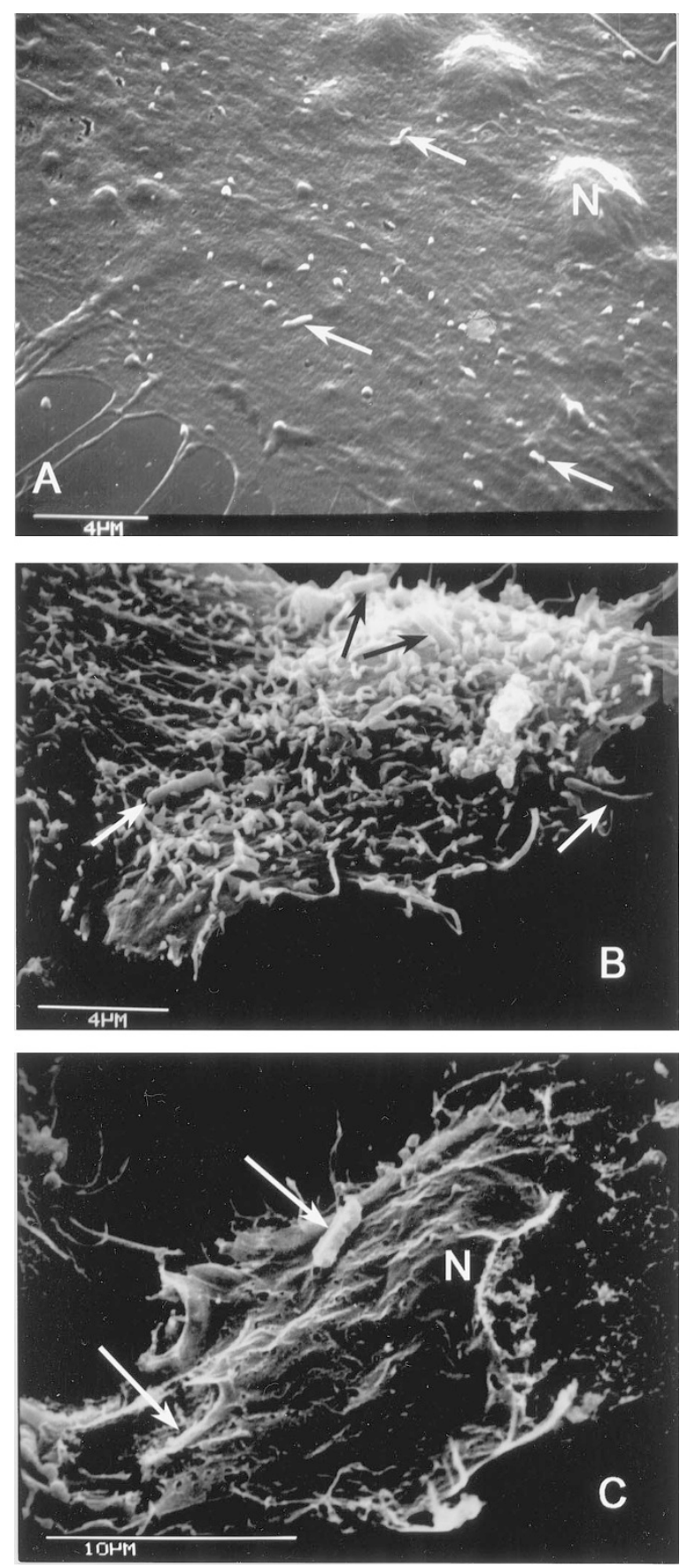

Figure 2.

By scanning electron microscopy, $M$. leprae binding to the HUVEC surface at $\mathrm{MOI}$ of 100:1 were easily observed at 3 hours $(A)$, and 6 hours $(B)$, and were present but less evident at 24 hours $(C)$. ( $N$ indicates HUVEC nuclei; arrows point to M. leprae)

leprae initially, but that these are rapidly ingested after binding. Over the next 24 hours of co-cultivation, increasing numbers of HUVEC participate in the binding and ingestion of $M$. leprae, again with rapid ingestion after binding, because bacilli do not appear to accumulate in large numbers on the cell surface. Even at $\mathrm{MOI}$ of 100:1, the majority of infected HUVEC have only ingested a small number of bacilli by 24 hours. Finally, if a very large bacterial load is presented
$(\mathrm{MOI}=1000: 1)$ nearly all cells will ingest at least some bacilli.

Not all pathogenic organisms are phagocytosed by EC (Filler et al, 1995) and $M$. leprae appears to be handled very slowly compared with other organisms that are. Maximal adherence of Borrelia burgdorferi to HUVEC was observed at 4 hours (Thomas and Comstock, 1989), for example, and uptake of Rickettsia rickettsii was maximal within 6 hours, infecting $80 \%$ to 90\% of HUVEC (Silverman, 1984; Walker, 1984).

Candida albicans and Listeria monocytogenes are capable of direct invasion of EC by means of proteins on their surfaces. Candida albicans adhere to EC in large numbers within 1 hour and rapidly penetrate them without phagocytosis (reviewed in Hostetter, 1994). Listeria monocytogenes is similarly capable of rapid binding to and invasion of EC (Drevets et al, 1995; Greiffenberg et al, 1998). No evidence is available at this time to indicate that $M$. leprae is capable of direct invasion by such mechanisms.

The impact of $M$. leprae infection on EC remains to be determined. Ingestion of some other organisms by EC is known to impair the barrier function of EC (Szczepanski and Benach, 1991) or to cause lethal damage to the host cell (Filler et al, 1995), but $M$. leprae is well known to be a very non-toxic intracellular parasite of macrophages and Schwann cells. Notably, under the conditions used in these studies, infection rates appeared to decline after 24 hours. This may be due to the death or preferential detachment of infected cells, but could also be an artifact of the culture conditions used in these studies.

Infection of EC may be an important factor in the pathogenesis of leprosy of all types. Infected epineural and perineurial EC appear to constitute an important reservoir of $M$. leprae on the surface of peripheral nerves (Scollard et al, 1999), greatly increasing the possibility of a high frequency and density of localized bacillemia within the nerves, as compared with other organs. Colonization of the vasculature of the epineurium may also increase the risk of ischemia due to even mild physiologic or mechanical stress. In addition, vasculitis is the requisite second half of the prevailing understanding of the erythema nodosum leprosum (ENL) reaction in leprosy —an immune complex vasculitis (Wemambu et al, 1969). Another rare but catastrophic reaction in lepromatous leprosy is the Lucio phenomenon -an acute, necrotizing vasculitis with high morbidity and mortality (Rea and Ridley, 1979; Scollard et al, unpublished data), which is also postulated to be mediated by immune complexes. EC may play important roles in the immunologic events in these acute reactions, either as an antigen-presenting cell or a target cell. To pursue these questions, detailed studies of the mechanisms of interaction between $M$. leprae and EC derived from different vascular beds will be required, because some important characteristics of EC differ depending on their origin. However, infection of HUVEC with $M$. leprae appears to offer a valuable system in which fundamental aspects of this interaction can be elucidated in vitro. 

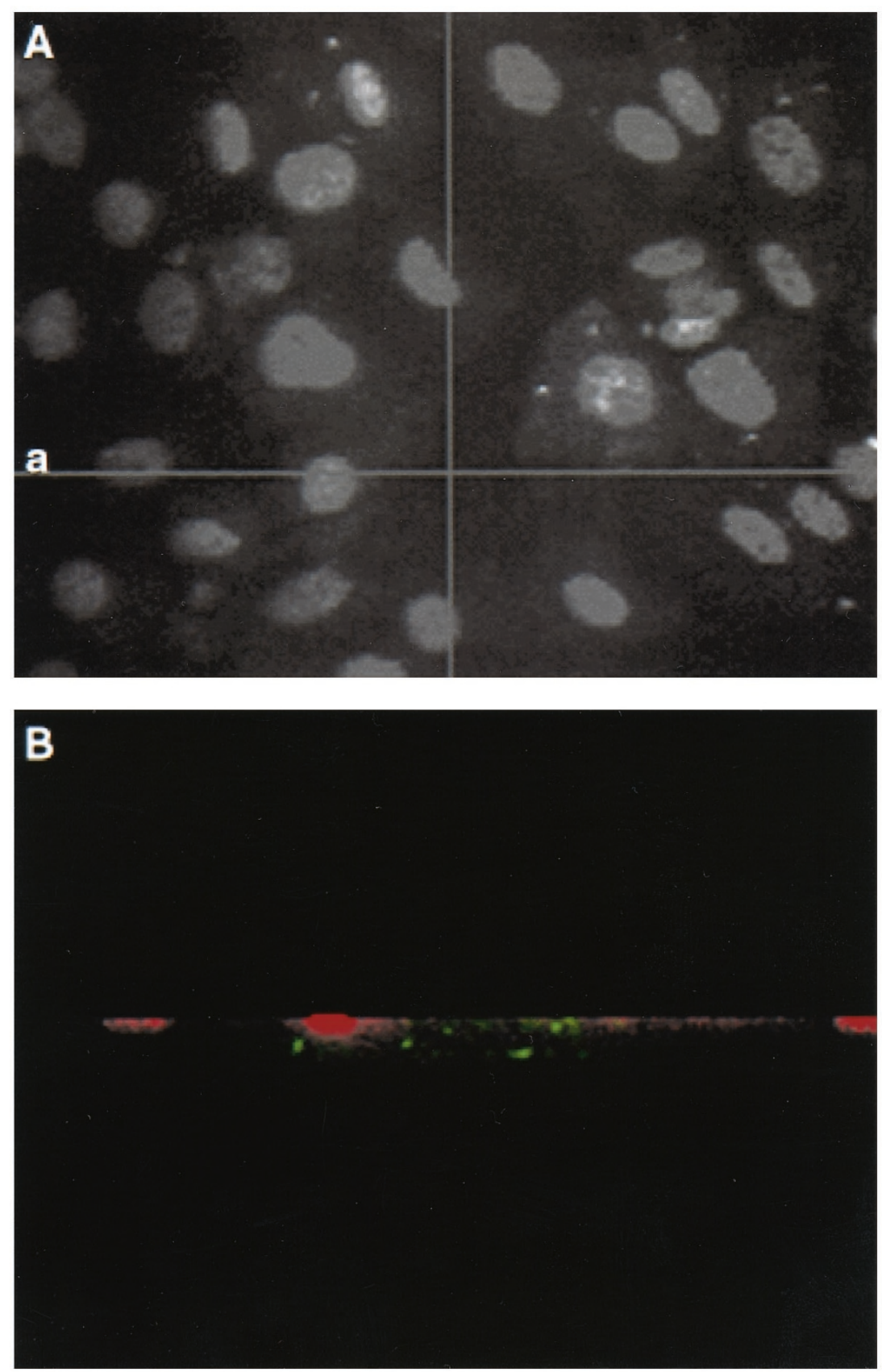

\section{Figure 3.}

Confocal laser scanning microscopy demonstrated internalization of $M$. leprae (24-hour culture) in cultures at $\mathrm{MOI}$ of $100: 1$. A, Original cumulative $x, y$ image of al layers at $550 \lambda$, showing HUVEC stained with TRITC-UEA-1. Cursor a provides orientation for $x, z$ analysis. B, Superimposed $x, z$ sections (at cursor a), at $550 \lambda$ and $515 \lambda$, colorized. Red indicates staining of HUVEC cytoplasmic and nuclear membranes with TRITC-UEA-1, at $550 \lambda$; Green indicates $M$. leprae stained with auramine- 0 , at $515 \lambda$.

\section{Materials and Methods}

\section{Cells and Culture Conditions}

HUVEC and a defined growth medium for these cells (containing $2 \%$ fetal bovine serum) were obtained from a commercial source (EGM, Clonetics, San Diego, California). Medium was changed every 48 to 72 hours, and cells were trypsinized and passaged approximately once per week. All experiments were performed using cells in passages 3 to 7 .
For experiments, HUVEC were cultured on uncoated, $12 \mathrm{~mm}$, round cell culture cover slips (Thermanox; Nalgene Nunc, Napierville, Illinois) in 24-well tissue culture plates, at $37^{\circ} \mathrm{C}$ in a humidified incubator with $10 \% \mathrm{CO}_{2}$. Round, $12 \mathrm{~mm}$ gelatin-coated glass coverslips were used for studies using confocal microscopy. Cells were seeded in $0.5 \mathrm{ml}$ medium, $10^{4}$ cells/well, and medium changed every 24 to 48 hours until they grew to confluence, usually 6 to 8 days. Fresh medium was applied at the time bacilli were added. 

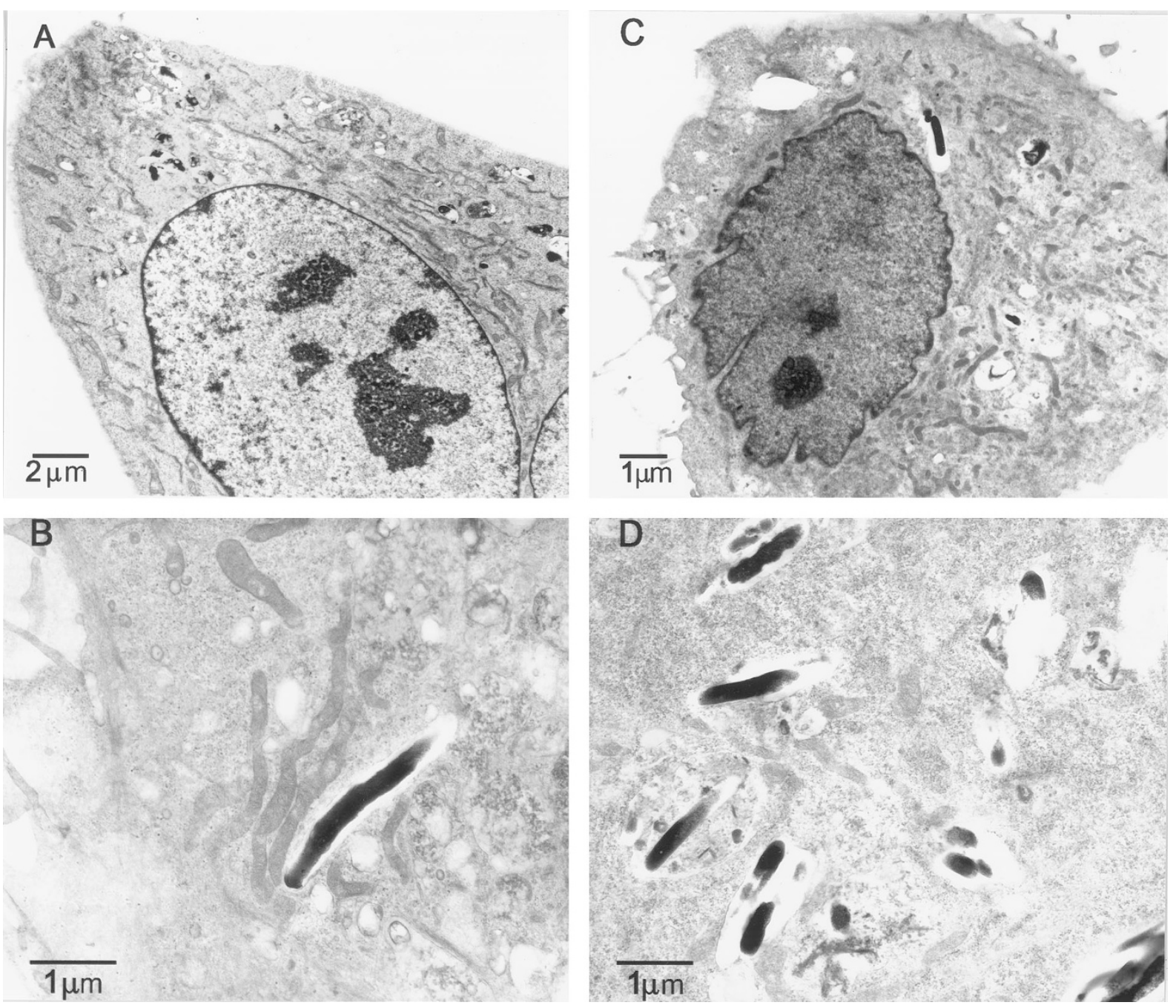

\section{Figure 4}

M. leprae were located inside phagosomes of HUVEC. Transmission electron microscopy demonstrated organisms inside phagocytic vacuoles within 1 hour (A and B), through 24 hours (C and D).

M. leprae. Bacilli were obtained from infected footpads of nude mice, harvested aseptically after 6 to 12 months, as previously described (Sibley and Krahenbuhl, 1988). Bacilli were tested free of contamination with other organisms, and possible tissue contaminants were reduced by incubation in $1 \mathrm{M} \mathrm{NaOH}$ for 10 minutes, followed by three washes in medium containing $10 \%$ fetal bovine serum. After overnight incubation at $4^{\circ} \mathrm{C}$, the concentration of bacilli was determined by counts of acid-fast organisms in calibrated smears, as previously described (Sibley and Krahenbuhl, 1988). Organisms were suspended in EGM medium, and added to cultures in a volume of $0.5 \mathrm{ml}$ per well at concentrations resulting in a multiplicity of infection (MOI) from 25:1 to 1000:1.

\section{Experimental Design}

After various intervals of culture, non-ingested bacilli were removed from the monolayer by washing each coverslip thoroughly, by dipping repeatedly in each of three changes of phosphate-buffered saline. After fixation in cold formol-acetone they were stained using a Kinyoun acid-fast stain and brilliant green counterstain ('BBL' TB Kinyoun Stain; Becton Dickinson, Towson, Maryland). The percentage of cells infected, and the bacillary load among infected cells, was determined by manual counting using a $100 \times$ objective under oil immersion. Cultures for each experimental condition were prepared in triplicate.

\section{Electron Microscopy}

For ultrastructural studies, monolayers on cover slips were washed as described above, and fixed in $1.25 \%$ glutaraldehyde and 2\% paraformaldehyde in $0.1 \mathrm{M}$ $\mathrm{Na}$-cacodylate buffer. After washing in the same buffer containing $5 \%$ sucrose, cells were post-fixed in $\mathrm{OsO}_{4}$ and dehydrated through a series of graded alcohols.

For scanning electron microscopy, specimens were processed in liquid $\mathrm{CO}_{2}$ in a Polaron critical point dryer (VG Microtech, West Sussex, United Kingdom), and coated with gold palladium (Hummer V sputter 
coater; Technics, San Jose, California). Specimens were examined with a Cambridge Stereoscan 150 scanning electron microscope (Leica, Deerfield, Illinois).

For transmission electron microscopy, fixed monolayers were embedded in Spurr resin and polymerized as previously described (Scollard et al, 1999). Embedded monolayers were detached from the cover slips, and ultrathin sections were cut parallel to the culture surface. Thin sections were collected on copper grids, stained with uranyl acetate and lead citrate, and examined under a Philips 410 electron microscope.

\section{Fluorescent Staining}

$M$. leprae were stained with $0.01 \%$ auramine-O (au-O; Sigma) (Truant et al, 1962) in EGM for 30 minutes at $37^{\circ} \mathrm{C}$. Specimens were then fixed in $1.25 \%$ glutaraldehyde at $4^{\circ} \mathrm{C}$ for 30 minutes, and washed in three volumes of PBS. The HUVEC surface membrane was stained with TRITC-conjugated Ulex europaeus-1 (rho-UEA-1) (Sigma), at a concentration of $300 \mathrm{mg} / \mathrm{ml}$ in PBS for 20 to 30 minutes at $4^{\circ} \mathrm{C}$. For dual staining with both reagents, specimens were first stained with au-O, fixed, and then stained with rho-UEA-1. Specimens were mounted face up in mounting medium composed of $50 \%$ glycerol containing p-phenylenediamine, $1 \mathrm{mg} / \mathrm{ml}$ (Platt and Michael, 1983), in a chamber on the glass slide created with two glass cover-slips on either side of the specimen to support another glass cover slip over the cells without crushing them (Bacallo et al, 1995). The margins were sealed with fingernail polish to prevent evaporation.

\section{Confocal Laser Scanning Microscopy}

Infected and uninfected monolayers were fixed, stained with rho-UEA-1, au-O, or both, as described above, and the specimens examined within 1 hour with a Noran Odyssey XL laser confocal instrument (Noran, Middleton, Wisconsin) using slit illumination, mounted on a Nikon microscope under $40 \times$ magnification, without oil. The image was collected $640 \times$ 480 resolution using Intervision acquisition software (Noran) and a Silicon Graphics Indy mini-workstation (Silicon Graphics, Houston, Texas). Images for each fluorochrome were collected in successive 0.3- $\mu$ layers, starting at the coverslip, using an excitation wavelength of $488 \lambda$ and a $515 \lambda$ long pass filter for Au-O, and excitation at $528 \lambda$ and a $550 \lambda$ long pass filter for rho-UEA-1. Data were analyzed in both $x, y$ and $x, z$ formats, and for $x, z$ evaluation, images were pseudocolorized using Adobe Photoshop software, and superimposed to demonstrate the relationship between the cytoplasmic membrane and $M$. leprae.

\section{Acknowledgements}

The author is grateful to J. P. Pasqua for preparation of M. leprae; Ron Bouchard of the Socolofsky Microscopy Center for assistance with confocal microscopy, G. McCormick and J. Allen for excellent technical assistance, and Penne Cason for assistance in preparation of the manuscript.

\section{References}

Austin FE, and Winkler HH (1988). Relationship of Rickettsial physiology and composition to the Rickettsia-host cell interaction. In: Walker DH, editor. Biology of rickettsial diseases. Boca Raton: CRC Press, 29-50.

Bacallo R, Kiai K, and Jesaitis L (1995). Guiding principles of specimen preservation for confocal fluorescence microscopy. In: Pawley JB, editor. Handbook of biological confocal microscopy, New York: Plenum Press.

Boddingius J (1984). Ultrastructural and histophysiological studies on the blood-nerve barrier and perineural barrier in leprosy neuropathy. Arch Neuropathol (Berl) 64:282-296.

Burchard PJ and Bierther M (1985). An electron microscopic study of small cutaneous vessels in lepromatous leprosy. Inter J Lepr 53:70-74.

Coruh G, and McDougall AC (1979). Untreated lepromatous leprosy: Histopathological findings in cutaneous blood vessels. Inter J Lepr 47:500-501.

Drevets DA, Sawyer RT, Potter TA, and Campbell PA (1995). Listeria monocytogenes infects human endothelial cells by two distinct mechanisms. Infect Immun 63:4268-4276.

Filler SG, Swerdloff JN, Hobbs C, and Luckett PM (1995). Penetration and damage of endothelial cells by Candida albicans. Infect Immun 63:976-983.

Fite GL (1941). The vascular lesions of leprosy. Inter J Lepr 9:193-202.

Fratti RA, Belanger PH, Ghannoum MA, Edwards JE, and Filler SG (1998). Endothelial cell injury caused by Candida albicans is dependent on iron. Infect Immun 66:191-196.

Gimbrone MA (1976). Culture of vascular endothelium. Progr Hemostasis Thrombosis 3:1-28.

Greiffenberg L, Goebel W, Kim KS, Weiglein I, Bubert A, Engelbrecht F, Stins M, and Kuhn M (1998). Interaction of Listeria monocytogenes with human brain microvascular endothelial cells: InIB-dependent invasion, long-term intracellular growth, and spread from macrophages to endothelial cells. Infect Immun 66:5260-5267.

Hansen GA, and Looft C. (1895). Leprosy in its clinical and pathological aspects. Translated 1973 by Norman Walker, Bristol. London: John Wright.

Hostetter MK (1994). Adhesions and ligands involved in the interaction of Candida spp. with epithelial and endothelial surfaces. Clin Microbiol Rev 7:29-42.

Klingmüller V (1930). Die lepra. Berlin: Springer Verlag, 535536.

Mukherjee A, Misra RS, and Meyers WM (1987). Endothelial cell bacillation in lepromatous leprosy: A case report. Lepr Rev 58:419-424.

Platt JL, and Michael AF (1983). Retardation of fading and enhancement of intensity of immunofluorescence by p-phenylenediamine. J Histochem Cytochem 31:840-842.

Rea TH, and Ridley DS (1979). Lucio's phenomenon: A comparative histological study. Inter J Lepr 47:161-166. 
Ryan US (1988). Phagocytic properties of endothelial cells. In: Ryan US, editor. Endothelial cells, vol 3. Boca Raton: CRC Press, 33-49.

Scollard DM, Lathrop GW, and Truman RW (1996). Infection of distal peripheral nerves by $M$. leprae in infected armadillos: An experimental model of nerve involvement in leprosy. Inter J Lepr 64:146-151.

Scollard DM, McCormick G, and Allen JL (1999). Localization of $M$. leprae to endothelial cells of epineural and perineurial blood vessels and lymphatics. Am J Pathol 154:1611-1620.

Sibley LD, Franzblau SG, and Krahenbuhl JL (1987). Intracellular fate of $M$. leprae in normal and activated macrophages. Infect Immun 55:680-685.

Sibley LD, and Krahenbuhl JL (1988). Induction of unresponsiveness to gamma interferon in macrophages infected with Mycobacterium leprae. Infect Immun 56:1912-1919.

Silverman DJ (1984). Rickettsia rickettsii-induced cellular injury of human vascular endothelium in vitro. Infect Immun 44:545-553.
Szczepanski A, and Benach JL (1991). Lyme borreliosis: Host responses to Borrelia burgdorferi. Micriobiol Rev 55:21-34.

Thomas DD, and Comstock LE (1989). Interaction of Lyme disease spirochetes with cultured eukaryotic cells. Infect Immun 57:1324-1326.

Truant JP, Brett WA, and Thomas W Jr (1962). Fluorescence microscopy of tubercle bacilli stained with auramine and rhodamine. Henry Ford Hosp Med Bull 10:287-296.

Turkel SB, Van Hale HM, and Rea TH (1982). Ultrastructure of the dermal microvasculature in leprosy. Inter J Lepr 50:164181.

Walker TS (1984). Rickettsial interactions with human endothelial cells in vitro: Adherence and entry. Infect Immun 44:205.

Wemambu SNC, Turk JL, Waters MFR, and Rees RJW (1969). Erythema nodosum leprosum: A clinical manifestation of the Arthus phenomenon. Lancet 2:933-935. 